\title{
Article
}

\section{Assessing the Attitude of Tampa Bay Youth toward HIV Self-Testing Kits}

\author{
Sherry Zhang ${ }^{1}$, Isabella Lopez ${ }^{1}$, Bernard Washington ${ }^{2}$, Brittney Gaudet ${ }^{1}$, Carina A. Rodriguez ${ }^{2}$ \\ and Lisa J. Sanders $2, *$ \\ 1 Morsani College of Medicine, University of South Florida, Tampa, FL 33612, USA; sherryz@usf.edu (S.Z.); \\ lopezi@usf.edu (I.L.); bgaudet@usf.edu (B.G.) \\ 2 Division of Pediatric Infectious Diseases, Morsani College of Medicine, University of South Florida, \\ Tampa, FL 33606, USA; bwashington@usf.edu (B.W.); crodrig1@usf.edu (C.A.R.) \\ * Correspondence: lisasanders@usf.edu
}

check for

updates

Citation: Zhang, S.; Lopez, I.;

Washington, B.; Gaudet, B.;

Rodriguez, C.A.; Sanders, L.J

Assessing the Attitude of Tampa Bay Youth toward HIV Self-Testing Kits. Trop. Med. Infect. Dis. 2021, 6, 111. https://doi.org/10.3390/ tropicalmed6030111

Academic Editors: Isolde Birdthistle, Gina Dallabetta and Sanyukta Mathur

Received: 4 May 2021

Accepted: 21 June 2021

Published: 25 June 2021

Publisher's Note: MDPI stays neutral with regard to jurisdictional claims in published maps and institutional affiliations.

Copyright: (c) 2021 by the authors. Licensee MDPI, Basel, Switzerland. This article is an open access article distributed under the terms and conditions of the Creative Commons Attribution (CC BY) license (https:// creativecommons.org/licenses/by/ $4.0 /)$.

\begin{abstract}
In adults, data support the utility and acceptance of home HIV testing; however, in youth, particularly in the US, this has not been well studied. In this exploratory study, we surveyed Tampa Bay youth aged 16-27 and attending sexual health clinics between 1 June and 31 June 2018 $(n=133)$ regarding attitudes and perceptions towards HIV self-testing. While most indicated the clinic over home when asked for preferred testing location, study population and subgroup analysis demonstrated a positive response (agree) to Likert-scale questions regarding the use of home HIV self-testing kits and negative responses (strongly disagree) to "would not use self-testing kit". There was a significant difference between genders in testing location preference $(p=0.031)$ for those respondents that specified gender $(n=123)$, with males more likely to prefer home testing than females. This study suggests an openness of youth towards HIV home testing that could help to expand the number of youth aware of their HIV status.
\end{abstract}

Keywords: youth; adolescents; HIV testing; attitude; self-testing

\section{Introduction}

Youth and young adults are a priority target population in terms of HIV diagnosis and prevention, as they represent $21 \%$ of all newly diagnosed HIV cases in the United States (US) [1,2]. The Centers for Disease Control's HIV Surveillance Report from 2020 demonstrates that while the overall rates of HIV diagnosis for persons aged 13-24 decreased from 2014 to 2018, the rate for ages 20-24 remained the second-highest rate of all age brackets at 27.9 people per 100,000 [1]. High infection rates and a lack of knowledge of infection status are important issues to address when working to prevent the continued spread of HIV in young people. In 2018, of the estimated 47,800 youth living with HIV in the United States (US), only about half were aware of their infection status [3]. For male adolescents that have sex with other males, only $11.5 \%$ have been tested for HIV [4]. In addition, youth with HIV are the least likely of any age group to be retained in care or to have a suppressed viral load, thereby increasing the risk of transmission to their sexual partners.

Compounding a lack of knowledge of HIV status, studies have documented numerous other health-related prevention challenges in youth. Nationwide, surveys of sexually active high school students show that $46.2 \%$ say they did not use condoms during their last sexual intercourse [4]. Of male students who have sex with men, 13.5\% confirmed having had four or more sexual contacts in their lifetime, increasing the likelihood of HIV transmission [4]. About $20 \%$ of sexually active students drank alcohol or reported other substance use prior to their most recent sexual intercourse, leading to a greater likelihood of having sex without protection [4]. Youth are also less likely to use pre-exposure prophylaxis as a prevention strategy for HIV due to barriers including cost, access, and stigma [1]. Finally, various socioeconomic challenges for youth such as low-income households, homelessness, 
incarceration, and being uninsured contribute to lack of access to HIV testing and care and make viral suppression difficult [1].

Routine HIV testing is important in maintaining the sexual, reproductive, and general health of patients. It is well recognized that HIV testing is foundational to HIV prevention strategies, yet barriers to the widespread testing of youth remain. Estimates suggest that only $22 \%$ of high-school-aged youth have been tested, a number that remained virtually unchanged from 2005 to 2013 despite recommendations for routine screening [5]. A recent study of youth showed that only about $60 \%$ of youth having sexual activity with a positive partner had been tested for HIV or knew their status, while only $40 \%$ of youth knew the HIV status of their sexual partner [6]. Common reasons youth cite for not being tested include never having been offered testing, not thinking they could be HIV positive, being upset at the idea of testing positive to find out their status, not wanting anyone to find out their test results, and difficulties with the testing process [7]. In-home HIV self-testing for youth and/or their partners is one possible way to overcome some of these barriers and increase youth testing rates.

In adult populations, the literature supports the idea that in-home HIV testing increases testing rates, leads patients to seek appropriate care, and bolsters prevention efforts $[8,9]$. However, the acceptability and outcomes of HIV self-testing interventions in US youth have not been well studied. Specifically, it is not known if the benefits observed in adults regarding prevention and linkage to care can be replicated in US youth. The recent COVID-19 pandemic and subsequent worldwide lockdowns led to increased concerns about the availability of HIV testing and HIV/AIDS treatment. In response, the World Health Organization recommended HIV self-testing as an additional method to increase HIV testing coverage and frequency [10]. Studies in Sub-Saharan Africa during these COVID-19 lockdowns found a high acceptance rate of HIV self-testing across various demographics; however, these studies did not include anyone younger than 18 [10]. The purpose of this study was to evaluate the efficacy and feasibility of implementing in-home HIV testing for US youth by surveying youth attitudes regarding this type of testing, with the intent to provide a foundation for future inquiries into the role of HIV self-testing among youth populations.

\section{Materials and Methods}

\subsection{Study Population, Variables, and Design}

Our observational cross-sectional survey study was conducted on youth between the ages of 16 to 27 years old presenting to four clinics associated with the University of South Florida (USF) Department of Pediatrics. Clinics included the Ybor Youth Clinic, a storefront clinic providing youth sexual health services to under-resourced youth located in a high HIV-incidence zip code; two pediatric HIV Clinics, one at St. Petersburg's All Children's Hospital and the other at the Children's Medical Service Lakeland Clinic; and USF Student Health Services, which provides on-campus health service to university students. Youth who presented to any of these clinics for HIV or STI testing or who were known to be HIV positive were given the opportunity to participate in the study. Survey participation was voluntary and verbal consent to participate was obtained by study personnel. Patients were informed that their answers were confidential, anonymous, and would not influence their course of care. The paper-based surveys exploring attitudes and perceptions about HIV self-testing were distributed during the clinic check-in process or during the risk-reduction portion of rapid HIV-testing visits. Data were collected from 1 June to 31 July 2018 and transcribed from the paper survey to an electronic file by study personnel. No identifiable information was collected or stored.

The survey consisted of 25 questions regarding patient demographics, sexual history, opinions on HIV self-testing, and perceived limitations to self-testing. Demographic questions included age, gender, sexual history, and STI/HIV history. One question specifically asked where they would prefer to have HIV testing done, in a clinic or at home. Other attitudes and preferences towards HIV self-testing (e.g., "I would use an in-home, HIV self- 
testing kit if provided and explained to me by my doctor", "I would encourage my partner or partners to contact or see a doctor if their result of an in-home test was positive for HIV") were rated on a 5-point Likert scale from 1 (strongly disagree) to 5 (strongly agree).

\subsection{Statistical Analysis}

Patients with unknown or undetermined data for certain categories and responses were excluded from univariate analyses for those respective variables. The dataset was subdivided into subsets based on gender, previous HIV testing, age, previous STI testing, previous positive STI history, and previous experience performing a self-test. Differences in continuous variables were assessed using Student's $t$ test with Cohen's $d$ as the calculation for the effect size [11]. Analysis of differences between demographic groups regarding inhome versus clinic testing was performed with chi-square tests of independence. A Fisher's exact test was utilized in cases where $n<5$ and effect size was determined using Phi $(\varphi)$, given that the analysis utilized $2 \times 2$ contingency tables [12]. Likert scale results were compared against demographic groups and analyzed using an independent-samples $t$ test. Statistical significance was defined as $p \leq 0.05$ for each statistical test, without adjustment for multiple testing. Data were analyzed using SPSS Version 24 (IBM Corporation, Armonk, NY, USA).

\section{Results}

Of 143 surveys that were conducted, 10 without Likert rankings about attitudes and preferences towards HIV self-testing were excluded ( 5 females, 5 males, average age 21.9). Of the remaining 133 surveys, participants were not required to respond to all of the other questions for their results to be analyzed. Regarding the number of participants from each clinic site, 121 completed surveys at the Ybor Youth Clinic, 2 surveys were completed at each of the HIV clinics, and 8 surveys were completed at Student Health Services. Participants were 21.2 years old on average, with a standard deviation of 2.3 years. Ages ranged from 16 to 27 years old. Seven participants were under the age of 18. A total of $63(51.2 \%)$ of participants who specified a gender identified as female. Eight respondents left gender blank and two misinterpreted the question and responded with their sexual orientation rather than gender. Of the 133 youth surveyed, 97 (72.9\%) reported ever having been tested for HIV and, of the 97 patients whose HIV status was known, 11 reported being HIV positive. A total of $70.2 \%$ reported having undergone STI testing. Of those that had received STI testing previously, 52.9\% had tested positive for either gonorrhea or chlamydia. Characteristics of the 133 patients whose surveys were analyzed are summarized in Table 1.

\subsection{Preferred Location and Method}

Regarding preferred testing location (home versus clinic) 38 participants preferred testing at home versus 81 who preferred testing in a clinic (Figure 1). Four respondents did not have a preference.

Of the youth who specified a gender $(n=123)$, there was a significant difference between genders in preference for testing location. Males were more likely than females to prefer testing at home, but both males and females expressed an overall preference for clinic testing. There was no statistical difference associated with previous HIV testing, previous STI testing, history of a previous STI, and previous use of an HIV self-testing kit and preference for testing location. Age was not statistically associated with a preference for testing location (Table 2). Of the 11 youth surveyed who were known to be HIV positive, none had ever used a self-test test kit. Four preferred at-home testing and seven preferred testing in a clinic setting, reflecting attitudes similar to those of the other respondents. 
Table 1. Characteristics of Patients Who Completed Survey.

\begin{tabular}{|c|c|}
\hline Clinic Site & \# Patients $(\%)$ \\
\hline Ybor Youth Clinic & $121(90.98 \%)$ \\
\hline USF Student Health & $8(6.02 \%)$ \\
\hline St. Petersburg HIV Clinic & $2(1.50 \%)$ \\
\hline CMS Lakeland HIV Clinic & $2(1.50 \%)$ \\
\hline \multicolumn{2}{|c|}{ Gender } \\
\hline Female & $63(47.37 \%)$ \\
\hline Male & $60(45.11 \%)$ \\
\hline No Answer & $8(6.02 \%)$ \\
\hline Answered Sexual Orientation & $2(1.50 \%)$ \\
\hline \multicolumn{2}{|c|}{ Sexual Orientation } \\
\hline Heterosexual & $78(58.64 \%)$ \\
\hline Bisexual & $10(7.52 \%)$ \\
\hline Gay Female & $9(6.77 \%)$ \\
\hline Gay Male & $31(23.31 \%)$ \\
\hline Other & $5(3.76 \%)$ \\
\hline \multicolumn{2}{|c|}{ \# Sexual Partners in Past Year } \\
\hline $0-2$ & $60(45.11 \%)$ \\
\hline $3-5$ & $44(33.08 \%)$ \\
\hline $6-10$ & $15(11.28 \%)$ \\
\hline$>10$ & $6(4.51 \%)$ \\
\hline No Answer & $8(6.02 \%)$ \\
\hline \multicolumn{2}{|c|}{ History of Previous STI } \\
\hline Yes & $53(39.85 \%)$ \\
\hline No & $48(36.09 \%)$ \\
\hline Never Tested & $31(23.31 \%)$ \\
\hline No Answer & $1(0.75 \%)$ \\
\hline
\end{tabular}

\subsection{Attitudes Towards Testing Use}

Figure 2 shows the statements that youth responded to and the Likert-scale responses to the home-testing questions included in the survey. The results show that a large majority of participants were open to the idea of in-home testing, with average responses in the 4-5 range ("agree" to "strongly agree") for the statements relating to using an in-home HIV self-testing kit, seeing a doctor regarding results, encouraging use of kits with partners, and being supported in using an in-home test. Ten participants did not respond to the last question on the survey regarding not using an in-home test and 29 were not sure of their response. However, of those that responded, 63/94 (67\%) disagreed or strongly disagreed with the statement that they would not use an in-home HIV test. 


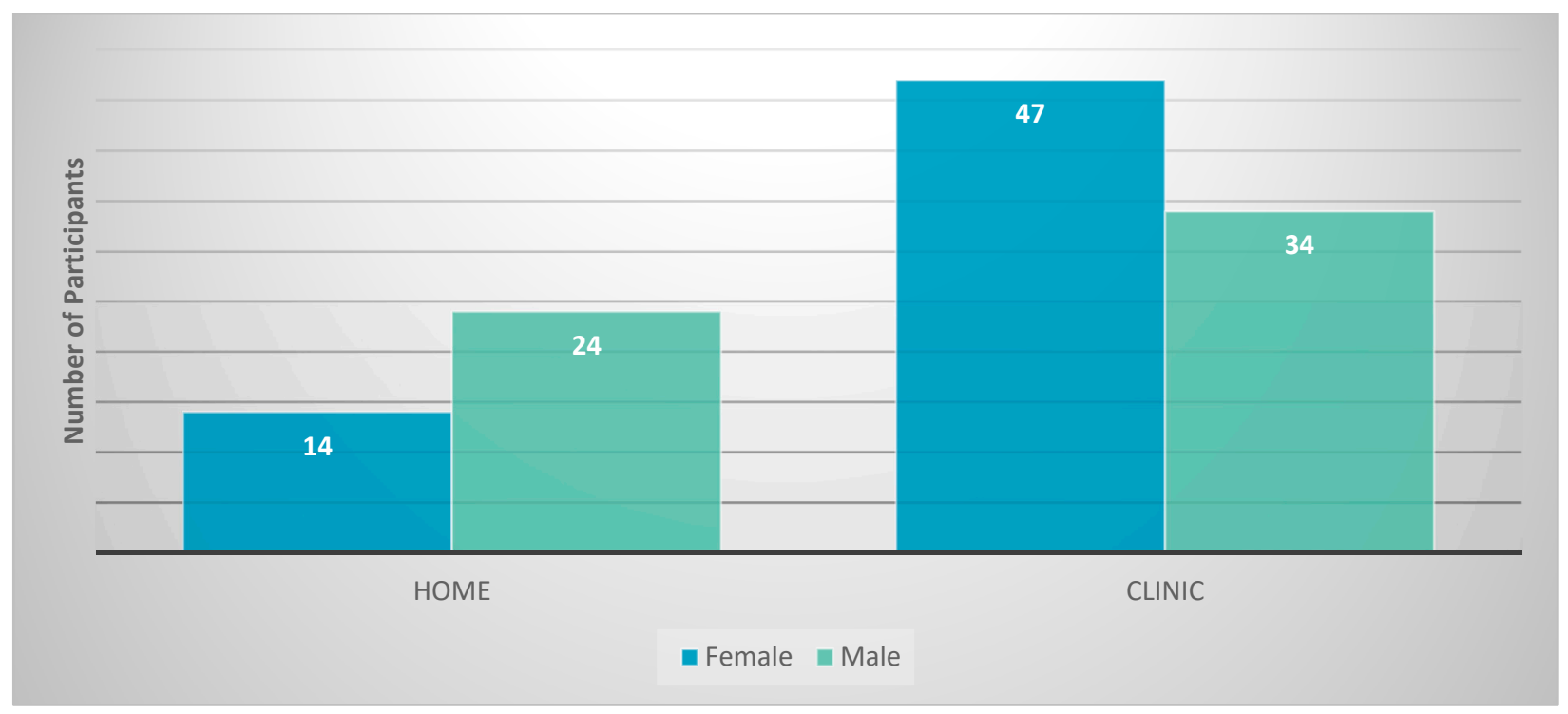

Figure 1. Testing location preference by gender.

Table 2. Demographics and Preference for Testing Location.

\begin{tabular}{|c|c|c|c|c|c|c|}
\hline & Home $(n=39)$ & Clinic $(n=90)$ & t or $x^{2}$ & $\mathrm{df}$ & $p$ & Effect Size \\
\hline Age & $20.49(2.29)^{1}$ & $21.27(2.26)^{1}$ & $\mathrm{t}=-1.790$ & 127 & 0.076 & 0.343 \\
\hline Gender & & & $\chi^{2}=4.645$ & 1 & 0.031 & 0.198 \\
\hline Female & $14(36.84 \%)$ & $47(58.02 \%)$ & & & & \\
\hline Male & $24(63.16 \%)$ & $34(41.98 \%)$ & & & & \\
\hline $\begin{array}{c}\text { HIV Test } \\
\text { Previously }\end{array}$ & & & $\chi^{2}=0.217$ & 1 & 0.641 & 0.060 \\
\hline No & $9(23.08 \%)$ & $26(28.89 \%)$ & & & & \\
\hline Yes & $30(76.92 \%)$ & $64(71.11 \%)$ & & & & \\
\hline $\begin{array}{c}\text { STI Test } \\
\text { Previously }\end{array}$ & & & $x^{2}=0.079$ & 1 & 0.779 & 0.025 \\
\hline No & $11(28.21 \%)$ & $27(30.68 \%)$ & & & & \\
\hline Yes & $28(71.79 \%)$ & $61(69.32 \%)$ & & & & \\
\hline $\begin{array}{c}\text { STI-Positive } \\
\text { Previously }\end{array}$ & & & $x^{2}=0.002$ & 1 & 0.968 & 0.004 \\
\hline No & $14(48.28 \%)$ & $33(47.83 \%)$ & & & & \\
\hline Yes & $15(51.72 \%)$ & $36(52.17 \%)$ & & & & \\
\hline $\begin{array}{c}\text { Ever Used } \\
\text { Self-Test Kit }\end{array}$ & & & _- & 1 & 1 & 0.019 \\
\hline No & 37 (97.37\%) & $86(96.63 \%)$ & & & & \\
\hline Yes & $1(2.63 \%)$ & $3(3.37 \%)$ & & & & \\
\hline
\end{tabular}

\footnotetext{
${ }^{1}$ Mean (standard deviation). Analysis excluded four youth without a preference for testing site and non-responders to the question.
}

There were no statistically significant differences $(p>0.05)$ between the various demographic groups (gender, previous HIV testing, previous STI testing, and ever STI positive) and responses to the Likert-scale questions regarding attitudes about HIV selftesting at home. HIV-positive patients were analyzed separately due to their positive status and possible differences in interpretation of the attitude questions, leading to measurement invariance. When doing subgroup analysis of the HIV-positive group, similar results were found regarding the Likert-scale questions. They averaged in the $4 \mathrm{~s}$ in regard to openness to home testing, and in the $1 \mathrm{~s}$ in response to not using an in-home HIV self-testing kit. 
I would use an in-home, HIV self-testing kit if provided and explained to me by my doctor.

I would contact my doctor if the resuts of an in-home selftest were positive for HIV.

I would see my doctor in person if the resuts of an in-home self-test were positive for HIV.

I would use an in-home HIV self-testing kit with my sexual partners.

I would encourage my partner or partners to contact or see a doctor if their result of an in-home test was positive for

HIV.

I would have support at home, from friends or family members, if I wanted to use an in-home HIV self-testing kit.

I would NOT use an in-home HIV self-testing kit.

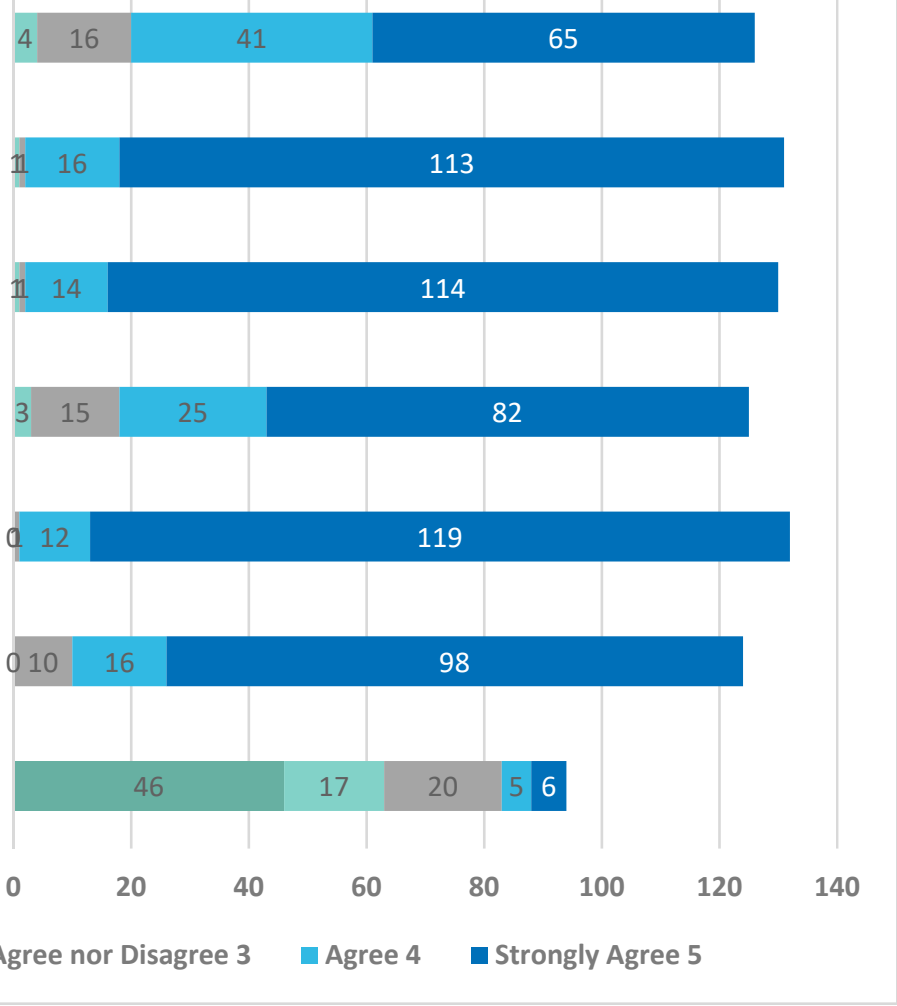

Figure 2. Likert-Scale Responses for HIV Self-Testing at Home Questions.

\section{Discussion}

A large hurdle for HIV prevention in youth is inconsistent testing leading to an unknown number of undiagnosed cases. A lack of awareness of infection status can lead to rapid spread of HIV infection. Youth engage in health-risking behaviors that lead to an increased susceptibility to HIV transmission, yet only approximately $59 \%$ of HIV-positive youth are aware of their infection [3]. This study sought to survey a young population at increased risk for HIV acquisition and transmission to assess the potential of expanding the use of HIV self-testing in this group. While our study was limited in scope, several interesting conclusions can be made from our results.

We chose to survey youth whose demographics indicated that they were likely to be at an increased risk of STI and HIV acquisition because these youth are the group most likely to benefit from HIV screening. Choosing to do our survey at clinics offering sexual health services was one way to allow us to narrow our focus to this group. Analysis of the demographics of the survey participants confirmed that they included youth who had multiple sexual partners, a past history of sexual transmitted infections, and young men who had sex with men.

When this group of youth at higher risk for STI and HIV acquisition were questioned about their preferred HIV-testing location, the majority reported a preference for testing in the clinic setting compared to home HIV testing. We did find a significant difference in testing location preference between genders, with males more likely than females to say they preferred home testing, but overall, higher numbers of males still indicated that they preferred clinic-based testing $(p=0.031)$. The fact that eight participants did not specify a gender and two misinterpreted this question suggest that this statistical finding may not be very robust and should be studied further. No statistical difference was noted regarding testing location preference between youth with previous HIV testing, previous STI testing, or a history of previous STI. In addition, there was no significant difference by age in preferred location of HIV testing. 
Despite the fact that youth initially reported a preference for testing in a clinic, their responses to the Likert-scale questions indicate an openness to considering self-testing. Overall, all of the youth studied were receptive to the idea of HIV self-testing, with broad agreement with questions involving a willingness to do self-testing and strong disagreement with the idea of never doing self-testing. There was no statistically significant difference $(p>0.05)$ using the Likert-scale questions regarding attitudes about HIV selftesting at home between gender, previous HIV testing, previous STI testing, previous STI diagnosis, or history of HIV positivity. This suggests that broad categories of youth, particularly those at higher risk of HIV acquisition, could be targets for HIV self-testing initiatives. While the youth we surveyed were very familiar with STI and HIV testing in the clinical setting, with over $70 \%$ having had previous testing, only four youth had performed a home test, which likely contributed to their preference for clinic testing. With appropriate educational efforts, our results support the idea that home HIV testing could be another useful tool to increase HIV testing rates in youth and encourage repeat testing in those with behaviors that continue to put them at risk for HIV acquisition.

There were several limitations to this study, including its exploratory nature. The data was gathered from a single geographical area, Tampa Bay, with potential for selection bias and not being representative of a larger population. The study population was not representative of the general population of US adolescents in terms of HIV-testing rate (72.9\% tested vs. 9-15\%), although it does represent a group that will benefit greatly from HIV screening; the results may not be generalizable to all youth. The responses were based on self-reporting data which are subject to recall and desirability biases. Other limitations are that the survey was not verified, some questions were not clearly understood by participants, and Likert questions could have been subject to both ceiling and floor effects.

We propose a larger multi-institutional study to both improve the power of the study and validate our findings. Further research should examine the discrepancy between genders in preference for location of testing to determine if this is due to our small sample size and omitted responses or reflects a true gender difference. We also did not address the impact of cost of self-testing kits on attitudes towards HIV self-testing, which should be explored in future research. Further studies to add to our understanding of youth attitudes regarding self-testing and testing location preferences will help to provide a foundation to develop approaches to increase testing rates in this population.

Author Contributions: Conceptualization, C.A.R., L.J.S. and B.W.; Methodology, C.A.R., L.J.S., B.W. and B.G.; Validation, C.A.R. and L.J.S.; Investigation, C.A.R., L.J.S., B.W. and B.G; Resources, C.A.R., L.J.S. and B.W.; Visualization, S.Z., I.L., C.A.R. and L.J.S.; Data Curation, S.Z. and B.G.; Formal Analysis, S.Z. and I.L.; Writing-Original Draft Preparation, S.Z., I.L., C.A.R. and L.J.S.; Writing-Review \& Editing, S.Z., I.L., C.A.R. and L.J.S.; Supervision, C.A.R. and L.J.S.; Project Administration, S.Z., I.L., C.A.R. and L.J.S. All authors have read and agreed to the published version of the manuscript.

Funding: This research received no external funding.

Institutional Review Board Statement: This study was reviewed by our institution's Scientific Review Committee and by our university's Institutional Review Board (Study \#001654) and determined to be a needs assessment to explore the possibility of incorporating home testing into clinical practices and as such was exempt as human subject research.

Informed Consent Statement: Patients gave verbal consent to complete the questionnaire although formal consent was waived by our institution's IRB based on the determination that this survey qualified as a needs assessment rather than human subjects research.

Data Availability Statement: The data presented in this study are available on request from the corresponding author.

Acknowledgments: We gratefully acknowledge Shannon Bailey, for her contributions to the statistical analysis. The authors would like to thank the staff of the Ybor Youth Clinic and the USF Pediatrics Clinics for allowing us to conduct our surveys at their clinics. Also, thank you to all of the clinic patients willing to share their opinions with us. 
Conflicts of Interest: The authors declare no conflict of interest.

\section{References}

1. Centers for Disease Control and Prevention. HIV Surveillance Report, 2018 (Updated); Vol. 31. Published May 2020. Available online: http:/ / www.cdc.gov/hiv/library/reports/hiv-surveillance.html (accessed on 3 July 2020).

2. United States Census Bureau. Age and Sex Composition. Available online: https://www.census.gov/library/publications/2011 /dec/c2010br-03.html (accessed on 10 June 2020).

3. Centers for Disease Control and Prevention. Estimated HIV Incidence and Prevalence in the United States, 2014-2018. HIV Surveillance Supplemental Report 2020; 25 (No. 1). Published May 2020. Available online: http://www.cdc.gov/hiv/library/ reports/hiv-surveillance.html (accessed on 3 July 2020).

4. Kann, L.; McManus, T.; Harris, W.A.; Shanklin, S.L.; Flint, K.H.; Queen, B.; Lowry, R.; Chyen, D.; Whittle, L.; Thornton, J.; et al. Youth Risk Behavior Surveillance-United States, 2017. MMWR. Surveill. Summ. 2018, 67, 1-114. [CrossRef] [PubMed]

5. Van Handel, M.; Kann, L.; Olsen, E.O.; Dietz, P. HIV Testing Among US High School Students and Young Adults. Pediatrics 2016, 137, e20152700-9. [CrossRef] [PubMed]

6. Straub, D.M.; Arrington-Sanders, R.; Harris, D.R.; Willard, N.; Kapogiannis, B.; Emmanuel, P.; Futterman, D.; Ellen, J.M. Correlates of HIV Testing History Among Urban Youth Recruited Through Venue-Based Testing in 15 US Cities. Sex. Transm. Dis. 2011, 38, 691-696. [CrossRef] [PubMed]

7. Peralta, L.; Deeds, B.G.; Hipszer, S.; Ghalib, K. Barriers and Facilitators to Adolescent HIV Testing. AIDS Patient Care STDs 2007, 21, 400-408. [CrossRef] [PubMed]

8. Kuecuekbalaban, P.; Rostalski, T.; Schmidt, S.; Muehlan, H. Psychological, situational and application-related determinants of the intention to self-test: A factorial survey among students. BMC Health Serv. Res. 2017, 17, 1-12. [CrossRef] [PubMed]

9. Katz, D.A.; Golden, M.R.; Hughes, J.P.; Farquhar, C.; Stekler, J.D. HIV Self-Testing Increases HIV Testing Frequency in High-Risk Men Who Have Sex With Men: A Randomized Controlled Trial. JAIDS J. Acquir. Immune Defic. Syndr. 2018, 78, 505-512. [CrossRef] [PubMed]

10. Mhango, M.; Chitungo, I.; Dzinamarira, T. COVID-19 Lockdowns: Impact on Facility-Based HIV Testing and the Case for the Scaling Up of Home-Based Testing Services in Sub-Saharan Africa. AIDS Behav. 2020, 24, 3014-3016. [CrossRef] [PubMed]

11. Field, A. Discovering Statistics Using SPSS, 3rd ed.; Sage Publications Ltd.: London, UK, 2009.

12. Kim, H.Y. Statistical notes for clinical researchers: Chi-squared test and Fisher's exact test. Restor. Dent. Endod. 2017, 42, 152-155. [CrossRef] [PubMed] 